\title{
Somatostatin receptor expression, tumour response, and quality of life in patients with advanced hepatocellular carcinoma treated with long-acting octreotide
}

\author{
J Cebon $^{*, 1}$ and Australasian Gastro-Intestinal Trials Group (AGITG) AG000 IH Investigators ${ }^{2}$ \\ 'AGITG Trial Coordinating Centre, NHMRC Clinical Trials Centre, University of Sydney, Locked Bag 77, Camperdown, NSW I450, Australia
}

\begin{abstract}
Octreotide may extend survival in hepatocellular carcinoma (HCC). Forty-one per cent of HCCs have high-affinity somatostatin receptors. We aimed to determine the feasibility, safety, and activity of long-acting octreotide in advanced HCC; to identify the best method for assessing somatostatin receptor expression; to relate receptor expression to clinical outcomes; and to evaluate toxicity. Sixty-three patients with advanced HCC received intramuscular long-acting octreotide $20 \mathrm{mg}$ monthly until progression or toxicity. Median age was 67 years (range 28-81 years), male $81 \%$, Child-Pugh A 83\%, and B 17\%. The aetiologies of chronic liver disease were alcohol (22\%), viral hepatitis (44\%), and haemochromatosis (6\%). Prior treatments for HCC included surgery (8\%), chemotherapy (2\%), local ablation (1 1\%), and chemoembolisation (6\%). One patient had an objective partial tumour response (2\%, $95 \% \mathrm{Cl} 0-9 \%$ ). Serum alpha-fetoprotein levels decreased more than $50 \%$ in four (6\%). Median survival was 8 months. Thirty four of 6 I patients (56\%) had receptor expression detected by scintigraphy; no clear relationship with clinical outcomes was identified. There were few grade 3 or 4 toxicities: hyperglycaemia (8\%), hypoglycaemia (2\%), diarrhoea (5\%), and anorexia (2\%). Patients reported improvements in some symptoms, but no major changes in quality of life were detected. Long-acting octreotide is safe in advanced HCC. We found little evidence of anticancer activity. A definitive randomised trial would identify whether patients benefit from this treatment in other ways.
\end{abstract}

British Journal of Cancer (2006) 95, 853-861. doi:I0.1038/sj.bjc.6603325 www.bjcancer.com

Published online 5 September 2006

(c) 2006 Cancer Research UK

Keywords: hepatocellular carcinoma; octreotide; scintigraphy; receptors, somatostatin; toxicity; feasibility study

Hepatocellular carcinoma (HCC) is a major cause of cancer death and morbidity worldwide (Johnson, 1996) It is frequently complicated by pre-existing chronic liver disease, limiting therapeutic options. While early disease can be managed by resection or local ablation, decreased hepatic reserve may limit the options for surgery or systemic therapy. Furthermore, response rates for systemic therapy are low: no single agent has a response rate higher than approximately $20 \%$, and none improves survival (Leung and Johnson, 2001). Multiagent therapy using cis-platinum, doxorubicin, 5-fluorouracil, or interferon-alpha may show higher response rates (Leung et al, 1999). However, because patients with HCC frequently have advanced liver disease and associated comorbidities, they are often unable to tolerate intensive and toxic treatment. On the basis that some HCCs have hormone receptors, hormonal approaches (including tamoxifen in a variety of doses) have been evaluated; however, none of these has shown a survival advantage over best supportive care (Chow et al, 2002).

\footnotetext{
*Correspondence: Dr J Cebon;

E-mail: jonathan.cebon@ludwig.edu.au

${ }^{2}$ For more details regarding Australasian Gastro-Intestinal Trials Group (AGITG) AG000 I H Investigators see Appendix A

Received 8 September 2005; revised 17 July 2006; accepted 24 July 2006; published online 5 September 2006
}

A small randomised trial conducted by Kouroumalis et al (1998) suggested that survival was longer in a group treated with shortacting octreotide than in an untreated control group (median survival 13 vs 4 months, 12 -month survival 56 vs $13 \%, P=0.002$ ). High-affinity somatostatin receptors capable of binding octreotide are present in approximately $40 \%$ of HCC, and not in adjacent liver. However, the numbers of high-affinity receptors are lower than reported for neuroendocrine tumours (Reubi et al, 1999). Somatostatin is a potent inhibitor of several growth factors and angioneogenesis (Florio et al, 2003); it also has immunemodulating properties (Lambrecht, 2001). Neuroendocrine and epithelial tumour cell growth can be inhibited in vitro and in vivo by the semisynthetic somatostatin analogue, octreotide (Lamberts et al, 1990). The long-acting depot form of octreotide (sandostatin long-acting release (LAR)) is formulated in microspheres, enabling treatment to be delivered by monthly intramuscular (i.m.) injection. It is currently used for the treatment of acromegaly (Freda, 2002) and functional neuroendocrine tumours (de Herder and Lamberts, 2002). Little information is available about its elimination kinetics in cirrhosis, although the short-acting form has been studied (Jenkins et al, 1998).

In patients with portal hypertension, octreotide has been used extensively in the management of acute variceal bleeding and other complications, where it reduces portal blood pressure in patients with poor liver function (Jenkins et al, 1997). Although the benefit 
observed by Kouroumalis et al (1998) may be a direct anticancer effect mediated by specific receptors on HCC cells, its effects on portal hypertension may also reduce the frequency of variceal bleeding. Octreotide therefore has the potential to affect survival by more than one mechanism.

We undertook a clinical trial to evaluate octreotide LAR in patients with unresectable HCC. The aims of this study were to assess the feasibility, safety, and activity of octreotide LAR in patients with advanced HCC before considering a definitive randomised phase III trial. Because clinical outcomes could potentially depend on somatostatin receptor status, we sought to evaluate the relationship between somatostatin receptor expression, objective response rate, and survival. The utility of noninvasive scanning as a predictor of response was examined by correlating octreotide scintigraphic scanning and biopsy data with survival. Receptor expression was evaluated by immunohistochemistry (IHC) and external scanning using labelled ligand (octreoscan). Since the study population was largely composed of people with impaired liver function and portal hypertension, plasma pharmacokinetic studies were also performed to assist in the overall assessment of the feasibility of using octreotide LAR in these patients.

\section{PATIENTS AND METHODS}

All patients had unresectable HCC, diagnosed either by histology or by the combination of typical findings on imaging and alphafetoprotein level (AFP) $>500 \mathrm{IU} \mathrm{ml}^{-1}$. World Health Organisation performance status was $0-2$ and no previous treatment with octreotide for HCC was permitted. Exclusion criteria included white blood count $<2.0 \times 10^{9} 1^{-1}$, platelets $<50 \times 10^{9} 1^{-1}$, haemoglobin $<10.0 \mathrm{gl}^{-1}$, serum creatinine $>0.15 \mathrm{mmoll}^{-1}$, bilirubin $>50 \mathrm{moll}^{-1}$, albumin $<25 \mathrm{gl}^{-1}$, and AST and ALT $>5$ times the upper limit of normal. Patients were also excluded if the prothrombin ratio (INR) was $>2.0$, if they were receiving concurrent antitumour treatment for HCC, or if they had uncontrolled ascites requiring paracentesis within 4 weeks, variceal bleeding in the previous month, prior radiation therapy to the only evaluable site of disease, or Child-Pugh class C cirrhosis. All patients gave informed consent and the protocol was approved by the institutional review board of each participating institution.

Treatment consisted of octreotide LAR $20 \mathrm{mg}$ by deep i.m. injection every 28 days. The planned duration of treatment was 12 months in the absence of disease progression, unacceptable toxicity, or withdrawal at the patient's or doctor's discretion. An extension phase allowed treatment to continue beyond 12 months on an individual basis at the discretion of the principal investigator.

Before starting treatment, all subjects had a complete medical history and physical examination, chest X-ray, and imaging to fully define the extent of disease. Triple-phase spiral computed tomography $(\mathrm{CT})$ was recommended for imaging the liver. Blood tests included full blood count, tests of liver function (serum albumin, alkaline phosphatase, total bilirubin, AST, and ALT), coagulation (INR and APTT), blood sugar, AFP, and tests to evaluate the aetiology of liver disease (HBsAg and anti-HCV). Blood was also sampled for octreotide pharmacokinetics and assessment of chromogranin A levels before the 4th and 7th doses. All patients had an octreotide scintigraphy scan before commencing treatment.

Tumour biopsy for receptor analysis was optional, depending on the availability of suitable tissue. Where available, archival material was assessed for receptor expression by IHC at a central laboratory.

Subjects were seen monthly for clinical review, blood tests, and administration of the study drug. Response was assessed using the
RECIST (response evaluation criteria in solid tumours) criteria (Therasse et al, 2000). Radiological assessment was undertaken 3monthly and any partial or complete responses were confirmed 1 month later.

Health-related quality of life (HRQL) was self-rated by patients each month before their clinical review using two instruments: the FACT-Hep (Heffernan et $a l, 2002$ ) and the 'Patient Disease And Treatment Assessment' form (Pt DATA Form). After the first month of treatment, patients also rated how aspects of their HRQL had changed, using a series of transition scales referred to here as the subjective Patient Benefit Form. We decided a priori to focus on changes in HRQL at 1 month because the number of evaluable patients was expected to fall quickly from then on.

The FACT-Hep (Heffernan et al, 2002) is a validated 45 -item self-rated questionnaire, incorporating the FACT-G questionnaire (27 items) (Cella et al, 1993), which covers multiple general aspects of HRQL, and an 18-item module specific for hepatobiliary cancer. Scores are calculated for four domains from the FACT-G: physical, social-family, emotional, and functional well-being. In addition, a total score including the hepatobiliary-specific items is calculated (Cella, 1997).

The Patient DATA Form is a simple, pragmatic, patient-rated instrument designed to measure aspects of HRQL that are relevant to people with advanced cancer. Its development is reported elsewhere (Nowak et al, manuscript in preparation). It assesses 24 aspects of HRQL using simply worded items listed on a single page: 16 physical and emotional symptoms of cancer rated on a numeric scale from 0 (no trouble at all) to 10 (worst I can imagine) and eight aspects of well-being rated from 0 (worst possible) to 10 (best possible) (Appendix A).

The Patient DATA Form is designed to be rapidly and easily interpreted: there is no scoring or aggregation procedure. Items are arranged in two blocks: symptoms and dysfunctions ( 0 on left, 10 on right) where high scores reflect worse quality of life or more severe symptoms; and aspects of well-being (10 on left, 0 on right) where high scores reflect better well-being and quality of life. Troublesome aspects stand out by being circled on the right side of the page.

The Patient Benefit Form is a health-transition scale that rates changes in the same aspects assessed with the Patient DATA Form using a similar simple, one-page format. Patients rated how each symptom or function has changed since their first injection 4 weeks previously using a five-point Likert scale: much better, a little better, the same, a little worse, or much worse.

\section{Pharmacokinetics}

Samples were taken immediately before the 4th and 7th doses of octreotide (weeks 12 and 24 of treatment). These time points were selected in order to assess trough octreotide plasma concentrations at steady state after three doses and to determine potential octreotide accumulation in this cirrhotic population with more continued dosing. Additional samples were also collected in some cases. Plasma octreotide levels were determined by radioimmunoassay kindly performed by $M$ Rouilly (Novartis Pharma AG, Basel, Switzerland). The lower limit of quantification was $50 \mathrm{pg} \mathrm{ml}^{-1}$ for a $50 \mu \mathrm{l}$ plasma sample.

\section{Octreotide scintigraphy}

${ }^{111}$ In-pentetreotide, $130-220 \mathrm{MBq}$, was administered intravenously. Images were acquired at all study sites with a standardised imaging protocol. All images were acquired on dual-headed gamma cameras using medium-energy parallel-hole collimators. Anterior and posterior planar images $\left(15 \mathrm{minimage}^{-1}\right)$ were acquired over the abdomen and at other sites of known disease $24 \mathrm{~h}$ after injection. Additional whole-body or SPECT images were acquired after 4 or $24 \mathrm{~h}$ in some patients. 
Two experienced nuclear medicine physicians assessed the images, blinded to each other's interpretation. Tracer uptake in the primary tumour and metastases was determined relative to normal liver. Abdominal CT scan reports were used for tumour localisation. Uptake at the tumour site was scored as negative, clear but faint, moderate, or intense. Disagreement between reviewers was resolved by consensus.

\section{Immunopathology}

Archival paraffin-embedded tissue was stained for somatostatin receptor, type SSTR2A, using rabbit polyclonal antiserum SS800 (Jomar Diagnostics, Adelaide, Australia) raised against amino-acid sequences 355-369 (ETQRTLLNGDLQTSI) of human, rat, and mouse SSTR2A receptor coupled to KLH. This antibody recognises the C-terminus of human, rat, and mouse SSTR2A receptor. Specificity of antibody binding was determined by blocking with cognate peptide. Positive control staining was determined using normal pancreas, islet cell, and other neuroendocrine tumours, where cytoplasmic and membrane staining of normal pancreatic islets was observed. Three-micron sections were pretreated with $10 \mathrm{~mm}$ sodium citrate, $\mathrm{pH} 6.0$, for $2 \mathrm{~min}$. Endogenous peroxidase was blocked with $3 \%$ hydrogen peroxide for $10 \mathrm{~min}$. Antiserum was applied at $1: 2000$ in $50 \mathrm{~mm}$ Tris- $\mathrm{HCl}, \mathrm{pH} 7.6,0.05 \%$ for $30 \mathrm{~min}$ at room temperature. Chromogen was DAB (DakoCytomation, Sydney, Australia).

\section{Chromogranin A}

Plasma chromogranin A levels were measured to see whether this marker of neuroendocrine differentiation provided a useful correlation with receptor expression, thereby serving as a simple blood test for receptor-bearing tumours. The levels were assayed by Network Pathology (Melbourne, Australia), using the Chromogranin A ELISA kit (DakoCytomation, Sydney, Australia).

\section{Statistical methods}

The sample size of 63 was designed to give $>80 \%$ power to detect an 8-month difference in median survival comparing the $40 \%$ patients expected to have a positive octreoscan $v s$ the remainder expected to have a negative octreoscan (estimated survivals 12 and 4 months, respectively, as per the treatment and control arms in the Kouroumalis study). Survival times were calculated from the day of registration. Survival curves were calculated using the method of Kaplan-Meier. The log-rank test was use to compare the relationship between receptor expression by scintigraphy and survival duration. Proportions were compared using $\chi^{2}$ tests. Quality-of-life results were described and analysed with simple descriptive and comparative methods. For each item in the Patient Benefit Form, we recorded the proportions of patients reporting that each aspect was much better, a little better, the same, a little worse, or much worse than before starting treatment, and used $\chi^{2}$ tests to compare the proportions of patients who reported feeling better $v s$ those who reported feeling worse. For the Patient DATA Form and FACT-Hep, we used Wilcoxon's rank-sum tests for paired data to compare baseline and 1-month scores for each domain. Responses from the Patient Benefit Form were compared with change scores from the Patient DATA Form using Spearman's rank-correlation coefficient.

\section{RESULTS}

The study accrued 63 patients with otherwise untreatable HCC between April 2001 and January 2002. Study sites are listed in Appendix A. The patients' baseline characteristics are shown in Table 1 . The median age was 67 years (range 28-81 years) and
Table I Patient characteristics

\begin{tabular}{|c|c|c|}
\hline Characteristic & $n$ & $\%$ \\
\hline \multicolumn{3}{|l|}{ Sex } \\
\hline Male & 51 & 81 \\
\hline Female & 12 & 19 \\
\hline \multicolumn{3}{|l|}{ WHO performance status } \\
\hline 0 & 20 & 32 \\
\hline I & 30 & 48 \\
\hline 2 & 13 & 21 \\
\hline \multicolumn{3}{|l|}{ Cirrhosis: Child_Pugh class } \\
\hline A & 52 & 83 \\
\hline B & 11 & 17 \\
\hline Raised AFP & 50 & 79 \\
\hline \multicolumn{3}{|l|}{ Race } \\
\hline Caucasian & 40 & 63 \\
\hline Asian & 14 & 22 \\
\hline African & । & 2 \\
\hline Polynesian or Melanesian & 4 & 6 \\
\hline Other & 4 & 6 \\
\hline \multicolumn{3}{|l|}{ Possible causes of $\mathrm{HCC}^{\mathrm{a}}$} \\
\hline Alcoholic liver disease & 14 & 22 \\
\hline Hepatitis & 40 & 63 \\
\hline $\mathrm{HBsAg}$ and no antibody to HCV & 16 & 25 \\
\hline Antibody to HCV and no HBsAg & 9 & 14 \\
\hline $\mathrm{HBsAg}$ and antibody to HCV & 3 & 5 \\
\hline No HBsAg or antibody to HCV & 6 & 10 \\
\hline Unclassified & 6 & 10 \\
\hline Haemochromatosis & 4 & 6 \\
\hline Other & 7 & 11 \\
\hline \multicolumn{3}{|l|}{ Most recent treatment for $\mathrm{HCC}$} \\
\hline No previous therapy & 31 & 49 \\
\hline Surgery & 5 & 8 \\
\hline Chemotherapy & I & 2 \\
\hline Local ablation & 7 & 11 \\
\hline Chemoembolisation & 4 & 6 \\
\hline Other & 15 & 24 \\
\hline
\end{tabular}

$\mathrm{HBs} A g=$ hepatitis $B$ surface antigen; $\mathrm{HCC}=$ hepatocellular carcinoma; $\mathrm{HCV}=$

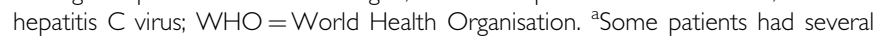
causes.

most of them were male. Most had good performance status. Eleven had Child-Pugh class B cirrhosis and 52 had wellcompensated disease (Child-Pugh class A). Viral hepatitis was the main cause of cirrhosis, with 28 patients (44\%) having evidence of hepatitis B or C infection. A small number of patients had cirrhosis from other causes. Approximately half had received other treatment for HCC.

\section{Administration of treatment}

Four patients $(6 \%)$ did not receive any octreotide because their disease progressed so rapidly they were unable to start treatment. These patients were not eligible for analysis of response or toxicity, but are included in the survival analysis. Three patients (5\%) received one dose, $12(19 \%)$ two doses, $10(16 \%)$ three doses, 10 (16\%) four doses, seven (11\%) five doses, four (6\%) six doses, five $(8 \%)$ seven doses, and seven (18\%) eight doses or more. The main reason for stopping treatment was disease progression in 19 patients $(30 \%)$. A further five patients $(8 \%)$ withdrew and nine (14\%) died during treatment, all from progressive disease. Treatment was stopped because of adverse effects (cramps) in only one case. 


\section{Tumour response}

Objective tumour response data for all 63 patients are summarised in Table 2. Reductions in AFP were accompanied by imaging evidence of partial response in one patient (2\%, 95\% CI 0-9\%), stable disease in one patient, and progressive disease in two patients.

\section{Survival}

Survival status is shown in Table 3 and Figure 1. After a median follow-up of 12 months, the median survival was 8 months (range

Table 2 Tumour responses

\begin{tabular}{|c|c|c|}
\hline Best response & $n$ & $\%$ \\
\hline \multicolumn{3}{|l|}{ Tumour response (RECIST) } \\
\hline Complete response & 0 & \\
\hline Partial response ${ }^{\mathrm{a}}$ & 1 & \\
\hline Stable disease & 15 & 24 \\
\hline Progressive disease & 23 & 37 \\
\hline Unknown & 9 & 13 \\
\hline Not available & 15 & 24 \\
\hline Total assessable & 63 & 100 \\
\hline \multicolumn{3}{|l|}{ AFP response $e^{\mathrm{a}, \mathrm{b}}$} \\
\hline$>50 \%$ reduction ${ }^{c}$ & 4 & \\
\hline$>50 \%$ reduction and no progressive disease ${ }^{c}$ & 2 & \\
\hline Total assessed & 59 & 100 \\
\hline
\end{tabular}

AFP = alpha-fetoprotein; RECIST = response evaluation criteria in solid tumours ${ }^{a}$ Confirmed on two consecutive assessments at least 4 weeks apart. ${ }^{\text {b Baseline AFP }}$ level needed to be more twice the upper limit of normal to qualify as a response. 'These two AFP responses without progressive disease are also counted in the row above.

Table 3 Survival status at March 2003

\begin{tabular}{lrr}
\hline Patient status & $\boldsymbol{n}$ & $\%$ \\
\hline Dead (tumour progression) & 48 & 76 \\
Dead (other causes) & 2 & 3 \\
Alive, still on study treatment & 5 & 8 \\
Alive, not on study treatment & 8 & 12 \\
Total & 63 & 100 \\
\hline
\end{tabular}

${ }^{a}$ One, pneumonia; one, sepsis.

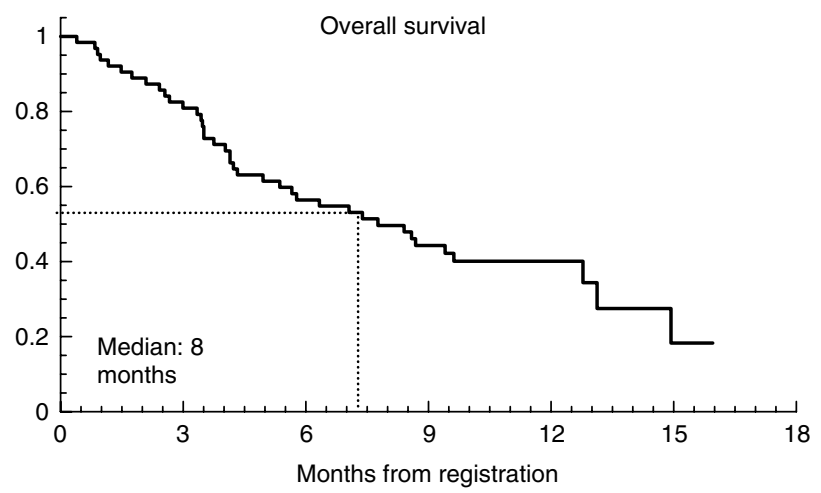

Number at risk

63

51

34

24

10

3

Figure I Overall survival.
1-25 months), and five patients remain on treatment 21-25 months after starting. There was no statistically significant relationship between receptor expression by octreotide scintigraphy and survival duration $(P=0.33)$ (Figure 2$)$.

Treatment was well tolerated. Table 4 shows the main toxicities, including diarrhoea, abdominal cramping, and alterations in blood sugar (chiefly hyperglycaemia). Most toxicities were grade 1 or 2; the only grade 3 or 4 toxicities were diarrhoea, alterations in blood sugar, and anorexia. Diarrhoea is a common symptom in patients with advanced HCC even in the absence of any treatment.

\section{Adverse events}

Most of the adverse events were attributable to chronic liver disease and its complications (Table 5). All deaths were attributable to progressive HCC. Two adverse events had unrelated causes (incarcerated inguinal hernia and an abscess).

\section{Gastrointestinal bleeding}

With a total follow-up approaching 4000 patient-months, gastrointestinal bleeding occurred in four of 63 patients. In one patient, this was from peptic ulcer disease and in the others was from varices.

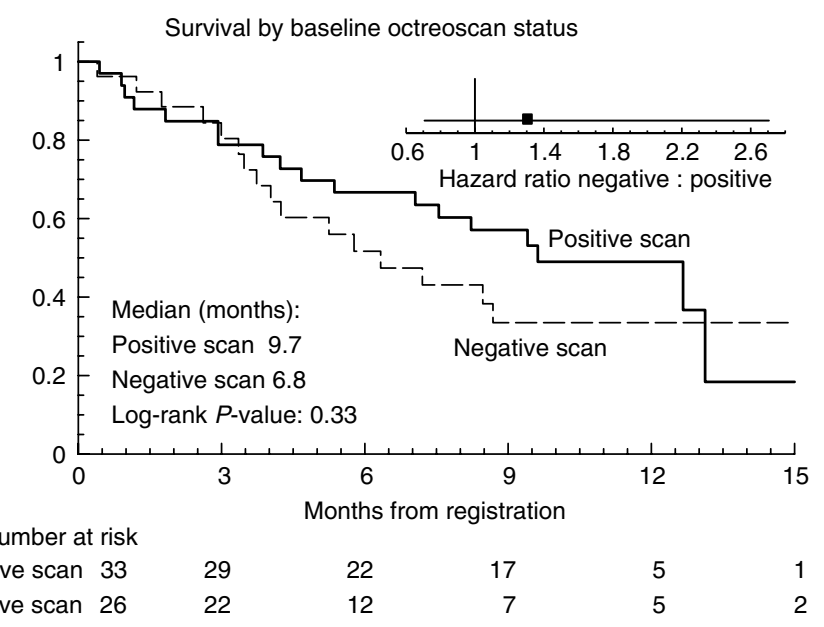

Figure 2 Survival, by baseline octreotide scintigraphy status. The small graph shows the hazard ratio of the negative group $(-)$ compared with the positive group $(+)$.

Table 4 Toxicity: numbers of patients with each grade as their most severe during their course of treatment

\begin{tabular}{lrrrrrr}
\hline & \multicolumn{5}{c}{ Toxicity grade $(\mathbf{N C l})^{\mathbf{a}}$} & \\
\cline { 2 - 5 } Type of toxicity & $\mathbf{0}^{\mathbf{b}}$ & $\mathbf{1}$ & $\mathbf{2}$ & $\mathbf{3}$ & $\mathbf{4}$ & \% with grade 3 or 4 \\
\hline Diarrhoea & 28 & 22 & 10 & 3 & 0 & 5 \\
Abdominal cramping & 47 & 9 & 7 & 0 & 0 & 0 \\
Hyperglycaemia & 45 & 4 & 9 & 5 & 0 & 8 \\
Hypoglycaemia & 60 & 2 & 0 & 0 & 1 & 2 \\
Bruising & 60 & 3 & 0 & 0 & 0 & 0 \\
Alopecia & 62 & 1 & 0 & 0 & 0 & 0 \\
Pancreatitis & 63 & 0 & 0 & 0 & 0 & 0 \\
Anorexia & 40 & 14 & 8 & 1 & 0 & 2 \\
Other & 63 & 0 & 0 & 0 & 0 & 0 \\
\hline
\end{tabular}

${ }^{\mathrm{a}} \mathrm{NCl}$ Common Toxicity Criteria Version 2. ${ }^{\mathrm{b}} \mathrm{Grade} 0=$ no toxicity. 


\section{Pharmacokinetics, receptors, and markers}

Pharmacokinetic evaluation was performed on $62,41,25$, and 7 patients at baseline and after 3, 6, and 9 months of treatment, respectively. The mean trough plasma concentration at 3 months was $2205 \mathrm{pg} \mathrm{ml}^{-1}$ (range 678-6166 $\mathrm{pg} \mathrm{ml}^{-1}$ ). After 6 months, the mean concentration of plasma octreotide was $3377 \mathrm{pg} \mathrm{ml}^{-1}$ (range 956-14290 $\mathrm{pg} \mathrm{ml}^{-1}$ ), and after 9 months, $3238 \mathrm{pg} \mathrm{ml}^{-1}$ (1307$9926 \mathrm{pg} \mathrm{ml}^{-1}$ ). Evaluation of those patients who remained on the study for 6 months or more showed no evidence of accumulation of octreotide in plasma. Although plasma concentrations fluctuated, they were generally within the range $1000-3000 \mathrm{pg} \mathrm{ml}^{-1}$.

Serum AFP was elevated in 50 patients $(79 \%)$. In four of these it fell, individually, from 578 to 2 , from 1600 to 2 , from 48 to 23 , and from 1368 to $56 \mathrm{IU} \mathrm{ml}^{-1}$. Serum for chromogranin A estimation and pharmacokinetics was available from 59 patients (94\%), and tissue was available for IHC analysis of somatostatin receptors from 20 samples from 19 patients (44\%); one patient had two tumours.

\section{Octreotide scintigraphy}

Sixty-one patients had octreotide scans. Table 6 shows the relationship between the degree of uptake in lesions as assessed by scintigraphy and the detection of tumour by conventional imaging. Overall, 34 of 61 patients (56\%) had positive scintigrams. Uptake was clear but faint in the majority of patients. Examples of octreotide uptake are shown in Figures 3 and 4. In general, the test was less sensitive than CT scanning in identifying HCC; however, it provided a noninvasive indication of the presence or absence of somatostatin receptors. In some instances (Figure 4), the tumour area showed less uptake than normal liver.

Table 5 Serious adverse events

\begin{tabular}{lr}
\hline Type of event & $\mathbf{n}$ \\
\hline Gastrointestinal bleeding & 4 \\
Ascites & 4 \\
Hepatic encephalopathy & । \\
Liver failure & । \\
Dyspnoea & । \\
Brain metastases & । \\
Diabetes mellitus & \\
Axillary abscess & 15 \\
Incarcerated hernia & \\
Total
\end{tabular}

\section{Immunohistochemistry for somatostatin receptors}

Positive controls (pancreatic islets, neuroendocrine tumours) showed clear staining. In the 20 tissue samples that were suitable for IHC analysis, there were not enough receptors for meaningful staining with the antiserum SS800, despite considerable efforts to optimise antibody staining.

\section{Chromogranin A}

Figure 5 shows chromogranin A levels. We hypothesised that patients with high levels might be those with tumours, which displayed features of neuroendocrine differentiation and therefore hormone receptors. In turn, these might be those most likely to respond to therapy with the somatostatin ligand. We therefore analysed the relationships among chromogranin levels, scintigraphy results, and clinical outcomes. There was no clear relationship between scan positivity and chromogranin A levels. Similarly, there was no relationship between serum levels of chromogranin A and survival.

\section{Quality of life}

Most patients had adequate English skills to complete the HRQL forms (47 patients, 75\%) and most did so at baseline (46 patients), but completing the forms decreased over follow-up, as expected. Fatigue, anxiety, pain, and insomnia were the symptoms rated as most severe at baseline. After 1 month of treatment, significantly more patients reported improvements than deteriorations on the Patient Benefit Form in vomiting, urinary symptoms, constipation, cough, irritability, and mood (Figure 6). Differences between ratings of these aspects at baseline and after 1 month, on the $\mathrm{Pt}$ DATA form, were in the same direction as the ratings from the Patient Benefit Form, but were not statistically significant, and correlations between the two instruments were modest (Spearman's $r<0.3$ ). There were no significant changes in the FACT-Hep between baseline and 1 month. More detailed analysis of the HRQL data will be reported elsewhere.

\section{DISCUSSION}

The main finding of this study was that octreotide LAR was well tolerated in patients with advanced HCC - a population with cirrhosis and chronic liver disease. Although there were few objective tumour responses (one patient with AFP response plus partial response on imaging, one patient with AFP response plus stable disease on imaging), 16 patients (25\%) had stable disease or better for at least 3 months. Forty per cent had only $1-3$ injections of octreotide LAR. It takes about 3 months for steady-state levels to be built up, so these patients were technically underdosed. The

Table 6 Octreotide scintigraphy (6I patients) and conventional imaging (CT scanning, 63 patients) compared

Number of patients with the given degree of radioactive uptake in the tumour at this site

\begin{tabular}{|c|c|c|c|c|c|}
\hline Site & $\begin{array}{l}\text { Patients with } \\
\text { disease at this } \\
\text { site confirmed } \\
\text { on } C T\end{array}$ & $\begin{array}{l}\text { Patients with } \\
\text { positive } \\
\text { scintigraphy at } \\
\text { this site }\end{array}$ & $\begin{array}{c}\text { Clear but } \\
\text { faint }\end{array}$ & Moderate & Intense \\
\hline Liver & 61 & 32 & 18 & 9 & 5 \\
\hline Lung & 3 & 0 & 0 & 0 & 0 \\
\hline Other & 11 & 4 & 3 & I & 0 \\
\hline
\end{tabular}

$\mathrm{CT}=$ computed tomography 

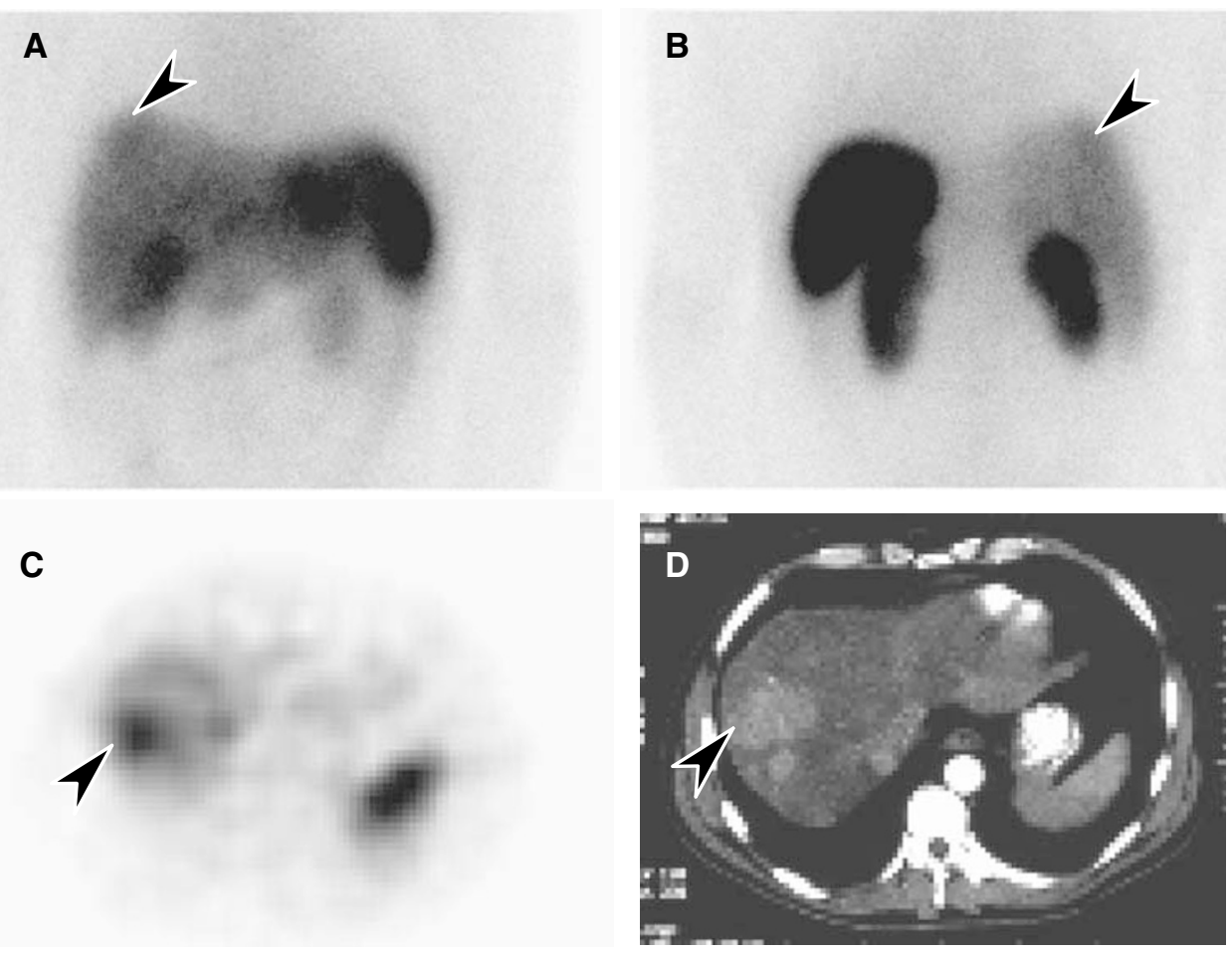

Figure 3 (A) Octreotide scintigraphy (4-h image) showing uptake in an HCC in the dome of the right lobe of the liver (arrow): anterior image; (B) posterior image; normal octreotide uptake in spleen and kidneys; (C) SPECT transaxial section through the upper abdomen showing increased uptake in the HCC (arrow); and (D) the hepatoma in a corresponding CT scan slice (arrow).
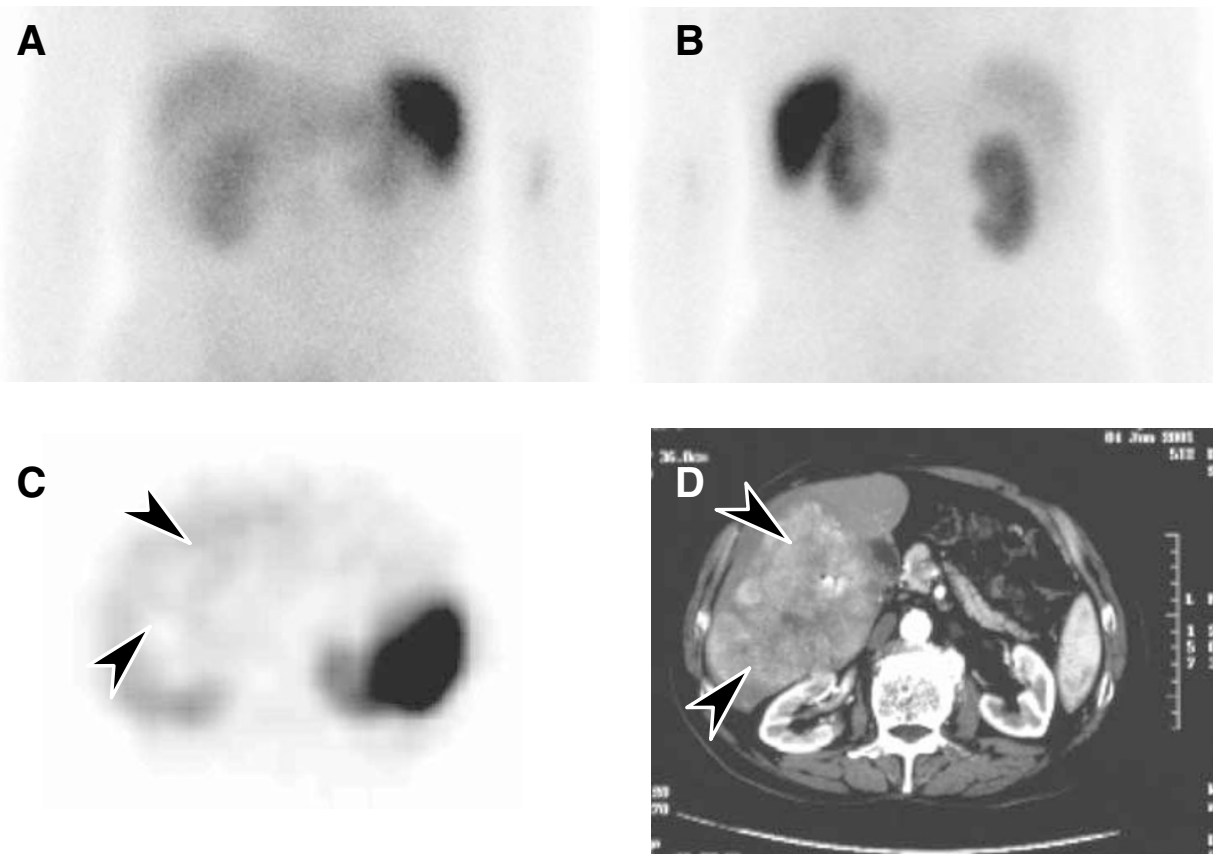

Figure 4 (A) Octreotide scintigraphy (4-h image) showing no evidence of uptake in an HCC in the right lobe of the liver: anterior image; (B) posterior image; (C) SPECT transaxial section through the upper abdomen showing reduced uptake in the HCC (arrows) compared with normal liver; and (D) the $\mathrm{HCC}$ in a corresponding CT scan slice (arrows).

median survival, 8 months, was within the range of published series (CLIP Group, 1998; Kouroumalis et al, 1998; Chow et al, 2002; Dimitroulopoulos et al, 2002; Samonakis et al, 2002; Yuen et al, 2002). Receptor expression was identified by octreotide scintigraphy in 34 of 61 patients (56\%). There was no clear relationship between scan positivity and survival. Approximately 
$25 \%$ of patients reported improvements in some aspects of HRQL while on octreotide.

Unresectable HCC commonly occurs in patients with advanced chronic liver disease and is difficult to treat. Comorbidities and poor performance status limit the use of cytotoxic chemotherapy, so identifying less toxic approaches is important. The small randomised trial reported by Kouroumalis in 1999 showed an implausibly large effect of octreotide on survival (Kouroumalis et al, 1998). It is impossible to tell from this trial whether this effect on survival was real, and if so, whether it was due to anticancer effects or other effects such as reduction in portal pressure. Subsequent studies with long-acting octreotide analogues have

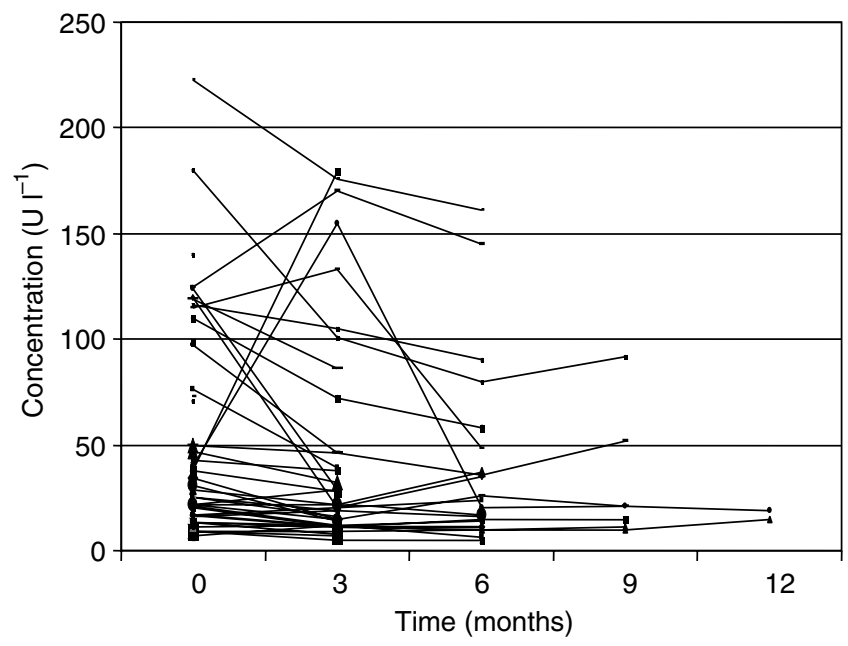

Figure 5 Chromogranin A levels over time in each patient. shown less benefit (Raderer et al, 2000; Samonakis et al, 2002) or no benefit (Yuen et al, 2002). In a recent study, patients selected for treatment on the basis of scan positivity were treated with octreotide and, when compared with a control population in a nonrandomised manner, had better outcomes in terms of survival and quality of life (Dimitroulopoulos et al, 2002). Nonetheless, this question will remain open until a definitive confirmatory randomised trial is performed.

Safety is important for people with comorbidities. There is limited information available about the elimination kinetics of octreotide in cirrhosis. Jenkins et al (1998) have demonstrated prolonged half-life and reduced clearance in patients with cirrhosis and suggested that dosage regimens should be modified in such patients to avoid accumulation of the analogue in the blood, which may result in undesirable side effects or toxicity. Since pharmacokinetics may be altered in patients with cirrhosis, it was considered necessary to determine whether liver impairment predisposes to toxicity and whether or not this dose was tolerated in this patient population. A somewhat reduced dose of $20 \mathrm{mg} \mathrm{month}^{-1}$ was arbitrarily selected to evaluate toxicity in this largely cirrhotic population. At this dose, plasma concentrations were achieved in the range $1000-3000 \mathrm{pg} \mathrm{ml}^{-1}$. These levels are similar to those typically seen in noncirrhotic patients. For example, a $30 \mathrm{mg}$ i.m. dose resulted in plasma concentrations of $1682 \pm 174 \mathrm{pg} \mathrm{ml}^{-1}$ in a series of six patients with acromegaly (Stewart et al, 1995). Although sufficient to suppress secretory function in acromegaly or carcinoid syndrome, it is unclear whether these concentrations are sufficient for therapeutic impact in HCC since a dose-response relationship has not been established for octreotide as an anticancer agent.

Octreotide treatment was well tolerated. The most common side effects were mild gastrointestinal symptoms and asymptomatic abnormalities of blood sugar levels. No serious adverse events were attributed to octreotide.

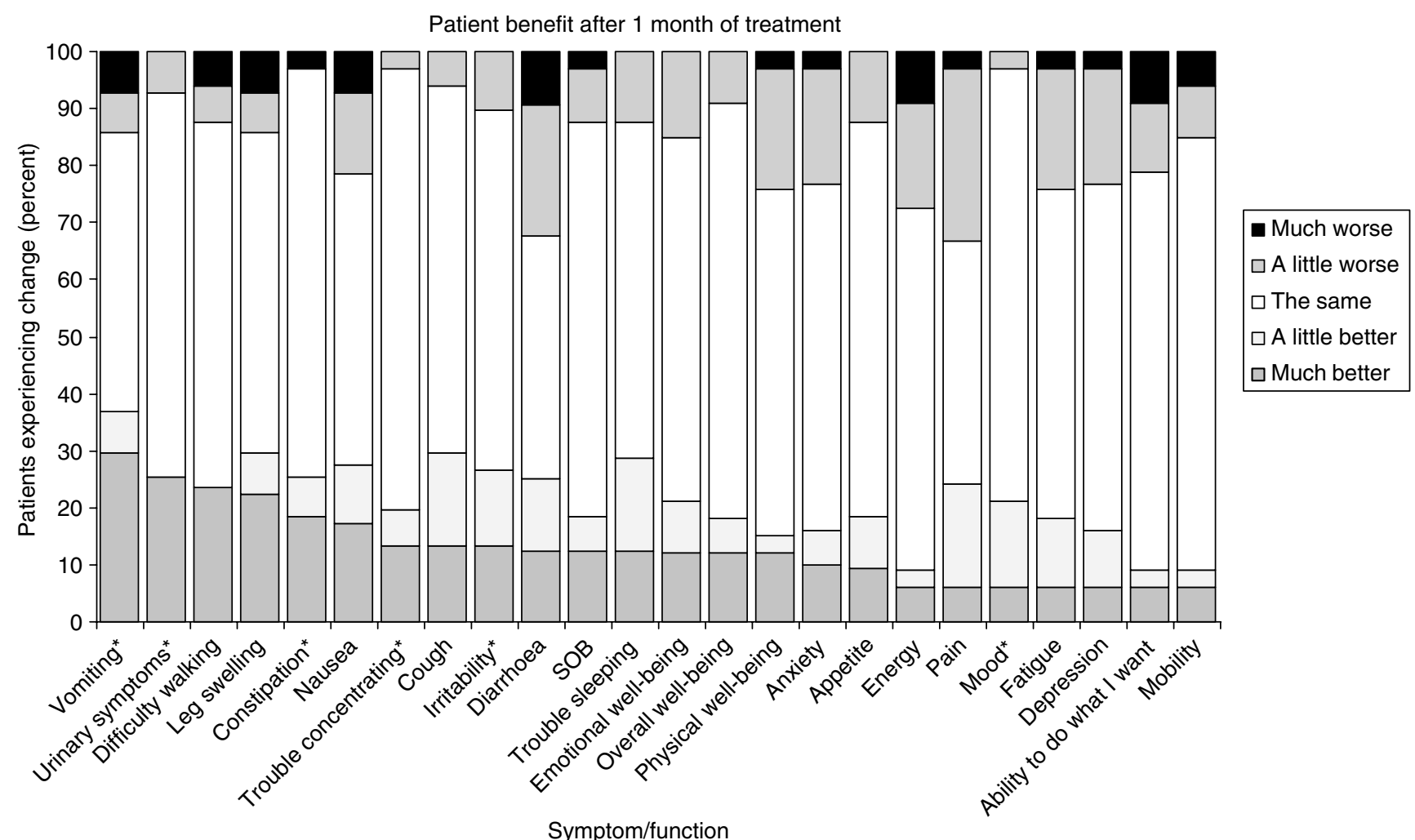

Figure 6 Subjective HRQL status reported on the PBF after I month of treatment. $* P<0.05$. 
Most patients with advanced HCC have a dismal prognosis (Leung and Johnson, 2001). However, a treatment that benefits only a subset of patients may still be clinically worthwhile. The very different outcomes in the randomised trials by Kouroumalis and Yuen probably reflect differences in patient selection (Kouroumalis et al, 1998; Yuen et al, 2002). The Yuen study had subjects with more advanced disease, a higher proportion with viral hepatitis, and a median survival of only 2 months. Our study population was more similar to the Kouroumalis study population (Table 4) and our median survival of 8 months lies midway between those of the two arms of the Kouroumalis study: 13 months in the octreotide arm and 4 months in the placebo arm.

As HCC can express high-affinity receptors for somatostatin (Reubi et al, 1999), it was important to determine whether a relationship existed between the presence of somatostatin receptors and clinical outcomes. We found that octreotide uptake was highly variable, ranging from less than the uptake of normal liver to high or intense (Table 6), and this variability was even seen within an individual (Figure 3). Few patients had intense uptake $(10 \%)$, consistent with the observation that receptor numbers in HCC tend to be lower than those in neuroendocrine tumours (Reubi et al, 1999). While octreotide scintigraphy was less helpful than CT scanning for identifying anatomical sites of tumour, its value may be in providing functional information about these tumours, particularly evidence of neuroendocrine differentiation. Confirmation of this using another technique is warranted. As fresh tissue was not consistently available, it was not possible to perform radioligand labelling studies to confirm this in vitro or at a microscopic level. Receptor analysis by IHC was performed on fixed tissues, but we were not able to reliably detect receptors in tissue sections. We were therefore unable to show a relationship between the antibody method and scintigraphy results.

Although HCCs do not typically show neuroendocrine differentiation on histological examination, measurement of serum chromogranin A levels was undertaken on the basis of a recent report that levels can be elevated in approximately one-third of cases (Leone et al, 2002). It was proposed as both a potential prognostic marker and a marker of neuroendocrine differentiation. In our series, six of 59 patients had levels $10 \mathrm{Ul}^{-1}$ or less (reference range $2-8 \mathrm{Ul}^{-1}$ ), 35 had levels of $10-50 \mathrm{Ul}^{-1}$, and 20 had levels higher than $50 \mathrm{Ul}^{-1}$. Although most patients progressed on radiological criteria, chromogranin A levels decreased in 15 of 59 patients $(25 \%)$ and remained stable in $25(40 \%)$, increasing in a sustained manner in only two patients (3\%). We found no evidence that receptor expression or neuroendocrine features were associated with either scan positivity or clinical outcomes: chromogranin A therefore did not appear to be a useful marker for identifying a subset of tumours more likely to respond, or as a marker for monitoring clinical progress, with the proviso that the small sample size did not provide enough statistical power to detect other than large effects.

Octreotide lowers portal pressure, and is routinely used to treat patients with variceal bleeding. Since prophylactic octreotide can prevent bleeding in high-risk patients (Jenkins et al, 1997), octreotide LAR might reduce morbidity and mortality through this mechanism. In our trial, gastrointestinal bleeding occurred in four patients (one peptic ulcer, three varices) with a total follow-up approaching 4000 patient-months. It is impossible to determine the effect of octreotide LAR on the rate of complications from portal hypertension without a randomly allocated control group; however, the rate in our study seems low compared with other series (Akanuma et al, 2002).

Although more patients rated improvements than deteriorations for some symptoms, these ratings were not strongly correlated with receptor status scores, and it is unclear whether these represent an effect of treatment. However, the fact that many patients reported improvements may partly explain the clinical impression of benefit in some patients. Determining the significance and cause of these changes requires a randomly allocated control group. Our findings support the feasibility and importance of incorporating measures of HRQL in future trials in advanced HCC.

This study has shown that octreotide LAR is well tolerated and may benefit some patients with HCC. We were unable to define, from octreotide scintigraphy or chromogranin A analyses, a subgroup more likely to benefit. There was little objective evidence of antitumour activity. A phase III study is advocated and indeed warranted given the conflicting findings with previous studies. However, careful attention should be paid to the selection of the primary end point and dose selection in the design of such a study. The potential impact on quality of life, survival, and cirrhosisrelated morbidity, particularly variceal bleeding, justifies the performance of such a trial.

\section{ACKNOWLEDGEMENTS}

This study was supported by Novartis Pharma AG.

\section{REFERENCES}

Akanuma M, Yoshida H, Okamoto M, Ogura K, Maeda S, Hata Y Sato S, Shiina S, Kawabe T, Shiratori Y, Omata M (2002) Risk factors for esophageal variceal bleeding in patients with hepatocellular carcinoma. Hepatogastroenterology 49(46): 1039-1044

Cella D (1997) Manual of the Functional Assessment of Chronic Illness Therapy (FACIT) measurement system (Version 4). Evanston: Northwestern Healthcare and Northwestern University, 1997

Cella DF, Tulsky DS, Gray G, Sarafian B, Linn E, Bonomi A, Silberman M, Yellen SB, Winicour P, Brannon J, Eckberg K, Lloyd S, Purl S, Blendowski C, Goodman M, Barnicle M, Stewart I, Mchale M, Bonomi P, Kaplan E, Taylor S, Thomas CR, Harris J (1993) The Functional Assessment of Cancer Therapy scale: development and validation of the general measure. J Clin Oncol 11: 570-579

Chow PK, Tai BC, Tan CK, Machin D, Win KM, Johnson PJ, Soo KC (2002) High-dose tamoxifen in the treatment of inoperable hepatocellular carcinoma: a multicenter randomized controlled trial. Hepatology 36(5): $1221-1226$

CLIP Group (1998) Tamoxifen in treatment of hepatocellular carcinoma: a randomised controlled trial. CLIP Group (Cancer of the Liver Italian Programme). Lancet 352(9121): 17-20

de Herder WW, Lamberts SW (2002) Somatostatin and somatostatin analogues: diagnostic and therapeutic uses. Curr Opin Oncol 14(1): $53-$ 57

Dimitroulopoulos D, Xinopoulos D, Tsamakidis K, Zisimopoulos A, Andriotis E, Markidou S, Panagiotakos D, Chrysohoou C, Bazinis A, Paraskevas E (2002) The role of sandostatin LAR in treating patients with advanced hepatocellular cancer. Hepatogastroenterology 49(47): 1245 1250

Florio T, Morini M, Villa V, Arena S, Corsaro A, Thellung S, Culler MD, Pfeffer U, Noonan DM, Schettini G, Albini A (2003) Somatostatin inhibits tumor angiogenesis and growth via somatostatin receptor-3-mediated regulation of endothelial nitric oxide synthase and mitogen-activated protein kinase activities. Endocrinology 144(4): 1574-1584

Freda PU (2002) Somatostatin analogs in acromegaly. J Clin Endocrinol Metab 87(7): 3013-3018

Heffernan N, Cella D, Webster K, Odom L, Martone M, Passik S, Bookbinder M, Fong Y, Jarnagin W, Blumgart L (2002) Measuring health-related quality of life in patients with hepatobiliary cancers: the functional assessment of cancer therapy-hepatobiliary questionnaire. J Clin Oncol 20(9): 2229-2239 
Jenkins SA, Baxter JN, Critchley M, Kingsnorth AN, Makin CA, Ellenbogen S, Grime JS, Love JG, Sutton R (1997) Randomised trial of octreotide for long term management of cirrhosis after variceal haemorrhage. $B M J$ 315(7119): $1338-1341$

Jenkins SA, Nott DM, Baxter JN (1998) Pharmacokinetics of octreotide in patients with cirrhosis and portal hypertension; relationship between the plasma levels of the analogue and the magnitude and duration of the reduction in corrected wedged hepatic venous pressure. HPB Surg 11(1): $13-21$

Johnson PJ (1996) The epidemiology of hepatocellular carcinoma. Eur J Gastroenterol Hepatol 8(9): 845-849

Kouroumalis E, Skordilis P, Thermos K, Vasilaki A, Moschandrea J, Manousos ON (1998) Treatment of hepatocellular carcinoma with octreotide: a randomised controlled study. Gut 42(3): $442-447$

Lamberts SW, Krenning EP, Klijn JG, Reubi JC (1990) The clinical use of somatostatin analogues in the treatment of cancer. Baillieres Clin Endocrinol Metab 4(1): 29-49

Lambrecht BN (2001) Immunologists getting nervous: neuropeptides, dendritic cells and T cell activation. Respir Res 2(3): 133-138

Leone N, Pellicano R, Brunello F, Rizzetto M, Ponzetto A (2002) Elevated serum chromogranin A in patients with hepatocellular carcinoma. Clin Exp Med 2(3): 119-123

Leung TW, Johnson PJ (2001) Systemic therapy for hepatocellular carcinoma. Semin Oncol 28(5): 514-520

Leung TW, Patt YZ, Lau WY, Ho SK, Yu SC, Chan AT, Mok TS, Yeo W, Liew CT, Leung NW, Tang AM, Johnson PJ (1999) Complete pathological remission is possible with systemic combination chemotherapy for inoperable hepatocellular carcinoma. Clin Cancer Res 5(7): 1676-1681

Raderer M, Hejna MH, Muller C, Kornek GV, Kurtaran A, Virgolini I, Fiebieger W, Hamilton G, Scheithauer W (2000) Treatment of hepatocellular cancer with the long acting somatostatin analog lanreotide in vitro and in vivo. Int J Oncol 16(6): 1197-1201

Reubi JC, Zimmermann A, Jonas S, Waser B, Neuhaus P, Laderach U, Wiedenmann B (1999) Regulatory peptide receptors in human hepatocellular carcinomas. Gut 45(5): 766-774

Samonakis DN, Moschandreas J, Arnaoutis T, Skordilis P, Leontidis C, Vafiades I, Kouroumalis E (2002) Treatment of hepatocellular carcinoma with long acting somatostatin analogues. Oncol Rep 9(4): 903-907

Stewart PM, Kane KF, Stewart SE, Lancranjan I, Sheppard MC (1995) Depot long-acting somatostatin analog (Sandostatin-LAR) is an effective treatment for acromegaly. J Clin Endocrinol Metab 80: $3267-3272$

Therasse P, Arbuck SG, Eisenhauer EA, Wanders J, Kaplan RS, Rubinstein L, Verweij J, Van Glabbeke M, van Oosterom AT, Christian MC, Gwyther SG (2000) New guidelines to evaluate the response to treatment in solid tumors. European Organization for Research and Treatment of Cancer, National Cancer Institute of the United States, National Cancer Institute of Canada. J Natl Cancer Inst 92(3): 205-216

Yuen MF, Poon RT, Lai CL, Fan ST, Lo CM, Wong KW, Wong WM, Wong BC (2002) A randomized placebo-controlled study of long-acting octreotide for the treatment of advanced hepatocellular carcinoma. Hepatology 36(3): 687-691

\section{Appendix A}

\section{Writing committee}

Jonathan Cebon

Michael Findlay

Carol Hargreaves

Martin Stockler

Paul Thompson

Michael Boyer

Stuart Roberts

Aurora Poon

Andrew M Scott

Victor Kalff

George Garas

Anthony Dowling

Darrell Crawford

John Ring

Russell Basser

Andrew Strickland

Graeme Macdonald

Michael Green

Anna Nowak

Blair Dickman

Haryana Dhillon

Val Gebski

Principal investigators

Paul Thompson

Jonathan Cebon

Michael Boyer

Stuart Roberts

George Garas
Austin Hospital, Melbourne

University of Auckland, Auckland

NHMRC Clinical Trials Centre, University of Sydney

NHMRC Clinical Trials Centre, University of Sydney

Auckland Hospital, Auckland

Royal Prince Alfred Hospital, Sydney

Alfred Hospital, Melbourne

Austin Hospital, Melbourne

Austin Hospital, Melbourne

Alfred Hospital, Melbourne

Sir Charles Gairdner Hospital, Perth

St Vincent's Hospital, Melbourne

Princess Alexandra Hospital, Brisbane

Flinders Medical Centre, Adelaide

Royal Melbourne Hospital, Melbourne

Monash Medical Centre, Melbourne

University of Queensland, Brisbane

Western Hospital, Melbourne

NHMRC Clinical Trials Centre, University of Sydney NHMRCClinical Trials Centre, University of Sydney NHMRC Clinical Trials Centre, University of Sydney NHMRC Clinical Trials Centre, University of Sydney

Auckland Hospital, New Zealand

Austin Hospital, Melbourne, Australia

Royal Prince Alfred Hospital, Sydney, Australia

Alfred Hospital, Melbourne, Australia

Sir Charles Gairdner Hospital, Perth, Australia
Anthony Dowling

Darrell Crawford

John Ring

Michael Findlay

Russell Basser

Andrew Strickland

Graeme Macdonald

Michael Green

St Vincent's Hospital, Melbourne, Australia Princess Alexander Hospital, Brisbane, Australia Flinders Medical Centre, Adelaide, Australia University of Auckland, New Zealand Royal Melbourne Hospital, Melbourne, Australia Monash Medical Centre, Melbourne, Australia University of Queensland, Brisbane, Australia Western Hospital, Melbourne, Australia

National Health and Medical Research Council Clinical Trials Centre, University of Sydney

Val Gebski and Carol Hargreaves (study statisticians)

Haryana Dhillon and Blair Dickman (study coordinators)

Anna Nowak and Martin Stockler (quality of life and clinical epidemiology)

Burcu Cakir (study manager)

Rhana Pike (publications editor)

\section{Trial Steering Committee}

Jonathan Cebon

Michael Findlay

Haryana Dhillon

Val Gebski

Garry Jeffrey

Graeme Macdonald

John Ring

Steve Riordan

Stuart Roberts

Andrew Scott

Martin Stockler

\author{
Austin Hospital \\ University of Auckland \\ NHMRC Clinical Trials Centre \\ NHMRC Clinical Trials Centre \\ Sir Charles Gairdner Hospital \\ University of Queensland \\ Flinders Medical Centre \\ Prince of Wales Hospital \\ Alfred Hospital \\ Austin Hospital \\ NHMRC Clinical Trials Centre
}

